\begin{tabular}{|c|c|c|}
\hline \multicolumn{2}{|c|}{ DJS Vol. 38 (2017) 7-18 } \\
\hline $\mathbf{1 9 6 9}$ & Delta Journal of Science & Available online at \\
\hline hesearch Article & https://djs.journals.ekb.eg/ & MATHEMATICS \\
\hline
\end{tabular}

\title{
Truncated Lindley Gamma Distribution
}

\author{
W.A.Hassanein ,T.A.El-Haddad \\ Department of Mathematics, Fac. of Science,Tanta University, Tanta, Egypt
}

\begin{abstract}
A new two-parameter truncated Lindley Gamma distribution (TLG) is proposed and its properties are studied. These properties include; the behaviour of TLG density function and hazard rate function, moments and the associated measures, reversed hazard function, mean residual life, Lorenz and Bonferroni curves. Point estimation of the parameters using the maximum likelihood and method of moments. Moreover, the interval estimation is investigated. Simulation schemes are carried out to examine the bias and mean square error of the estimators. Finally, a real data application illustrates the performance of TLG and compared with some well- known distributions.
\end{abstract}

Keywords: Truncation - Lindley distribution - Gamma Distribution.

\section{Introduction}

A lifetime distribution represents an attempt to describe, mathematically, the length of life of a system or device. With the advancement in technology, new and complex types of consumable items are being produced every day. To model the failure data of such types of new devices we need more plausible statistical distributions to be considered as lifetime and reliability models.

Numerous other parametric models have also been suggested and used in the analysis of lifetime data. Most of them are positively skewed statistical distributions with range restricted to positive part of the real line. Among others, exponential, gamma, Weibull and lognormal are most frequently used lifetime models. Pareto, Burr and half normal have also been used to model lifetime data. In addition, Lindley distribution is considered one of the famous distributions for lifetime data. It was first proposed by
Lindley [10] in connection with the fiducial distribution and Bayes theorem. It was compounded with Poisson distribution, by Lindley [9] and later by Shankaran [14]. In a recent study, Ghitany et al. [5] have discussed Lindley distribution in detail with its applications and real data example. For more detail of these distributions, see Lawless[7] Mann et al. [11], and Sinha[15].

In reliability studies a sample of $\mathrm{n}$ items is put on test and the experiments is terminated when all of them fail. This procedure may take a long time when the lifetime distribution of items has thick tail. Moreover, if the items are expensive such as medical equipment, it is costly to gather the whole sample information. There are many situations where experimental units are lost or removed from the test, before the complete failure. For example, the individuals in units may accidentally break. In other scenarios, the experiment may have to be terminated in order to free up testing facilities for other purposes. For these situations, the 
truncated distributions are quite effectively used where a random variable is restricted to be observed on some range and these situations are common in various fields.

Many researchers, therefore being involved to the problem of analyzing such truncated data encountered in various disciplines, proposed the truncated versions of the usual statistical distributions. Singh et. al.[16] introduced Lindley distribution with truncation. Ahmed et al. [2] discussed the application of the truncated version of the Birnbaum-Saunders (BS) distribution to improve a forecasting actuarial model and particularly for modelling data from insurance payments that establish a deductible. Aban et al. and Zaninetti et al. $[1,17]$ discussed the application of the truncated Pareto distribution to the statistical analysis of masses of stars and of diameters of asteroids. The truncated Weibull distribution has been found to apply in various fields such as to analyze the diameter data of trees truncate data specific threshold level, to model the diameter distribution of forest, to characterize the observed Portuguese fire size distribution, to seismological data, etc. For more detail on the truncated Weibull distribution and related references readers may refer to Murthy et al.[12] covered the subject of Weibull distribution and recently published article Zhang et al. [18] based on the truncated Weibull distribution.

This paper is organized as follows: Section 2 introduces the main structural prperities include; the probability density, cumulative distribution functions. In Section 3, the hazard, survival, mean residual life functions are derived and studied. Section 4 includes the moments and the associated measures of TLG. Characteristic and moment generating function are obtained in Section 5. Lorenz and Bonferroni curves are derived in Section 6 . Section 7 includes the point and interval estimation of TLG parameters. The order statistics is derived in Section 8. Section 9 includes the simulation study for TLG. Finally, a real life application is illustrated in Section 10 .

\section{Structural Properties}

\subsection{Probability density function}

A non-negative random variable $X$ is said to have the TLG distribution with parameters $\theta, \beta$, if its probability density function (pdf) is given by:

$$
f(x ; \theta, \beta)=\frac{\theta}{1+\theta} \text { Lindley }(\theta, \beta)+\frac{1}{1+\theta} \text { Gamma }
$$

$$
\begin{gathered}
=\frac{\theta}{1+\theta} \frac{\theta^{2}(1+x)}{\theta(1+\beta)+1} e^{-\theta(x-\beta)} \\
+\frac{1}{1+\theta} \theta^{2}(x-\beta) e^{-\theta(x-\beta)}
\end{gathered}
$$

$f(x ; \theta, \beta)=\frac{\theta^{2}}{1+\theta}\left((x-\beta)+\frac{\theta(1+x)}{\theta(1+\beta)+1}\right) e^{-\theta(x-\beta)} ; x>$

$\beta, \theta>0, \beta>0$

The behavior of the p.d.f. of TLG distribution at $x=\beta$ and at infinity are given by

$f(\beta)=\frac{(1+\beta) \theta^{3}}{(1+\theta)(1+(1+\beta) \theta)} \quad, \quad \lim _{x \rightarrow \infty} f(x)=\infty$

\section{Theorem 1.}

The p.d.f. $f(x)$ in (1) of TLG distribution is

(a)decreasing if $\theta>1, \beta>0, x>\beta$

(b)unimodal if $0<\theta \leq 1, \beta>0, x>\beta$

Proof.

The first derivative of $f(x)$ is obtained from(1) as:

$$
\begin{aligned}
f^{\prime}(\mathrm{x})=\left(e^{\wedge}((-x\right. & +\beta) \theta) \theta^{\wedge} 2(1-\theta(-2+x+\theta \\
& +x(2+\beta) \theta-\beta(2+\theta \\
& +\beta \theta)))) /((1+\theta)(1+\theta+\beta \theta))
\end{aligned}
$$

By solving $f^{\prime}(\mathrm{x})=0$, for $0<\theta \leq 1, \beta>0, x>$ $\beta$, the mode $x_{0}$ is

$$
x_{0}=\frac{1+2 \theta+2 \beta \theta-\theta^{2}+\beta \theta^{2}+\beta^{2} \theta^{2}}{\theta(1+2 \theta+\beta \theta)}
$$


Where, the second derivative of $f(x)$ is given by

$$
\begin{aligned}
f^{\prime \prime}(\mathrm{x})=\left(e^{\wedge}((-x\right. & +\beta) \theta) \theta^{\wedge} 3(-2+\theta(-4+x+\theta \\
& +x(2+\beta) \theta-\beta(3+\theta \\
& +\beta \theta)))) /((1+\theta)(1+\theta+\beta \theta))
\end{aligned}
$$

$$
\beta=0.01,0.1<\theta<0.5
$$

And $f^{\prime \prime}(\mathrm{x})$ at the point $x_{0}$ is :

$f^{\prime \prime}\left(x_{0}\right)=-\frac{e^{\frac{-1+\theta(-2+\beta(-1+\theta)+\theta)}{1+(2+\beta) \theta}} \theta^{3}(1+(2+\beta) \theta)}{(1+\theta)(1+\theta+\beta \theta)}<0$, then $f(x)$ has aglobal maximum at point $x_{0}$.Moreover,

$$
\begin{aligned}
\ln f(x)=2 \ln (\theta) & -\ln (1+\theta) \\
& +\ln ((x-\beta)(\theta(1+\beta)+1))+\theta(1 \\
& +x)) \\
& -\ln ((\theta(1+\beta)+1))-\theta(x-\beta)
\end{aligned}
$$

$\frac{d \ln f(x)}{d x}=\frac{1+2 \theta+\theta \beta}{(x-\beta)(\theta(1+\beta)+1)+\theta(1+x)}-\theta$

$\frac{d^{2} \ln f(x)}{d x^{2}}=-\left(\frac{1+2 \theta+\theta \beta}{(x-\beta)(\theta(1+\beta)+1)+\theta(1+x)}\right)^{2}$

Hence, $f(x)$ could attain a maximum, or a point inflection where $\frac{d^{2} \ln f(x)}{d x^{2}}<0$ and if $\theta>1, \beta>0$, $\frac{d \ln f(x)}{d x}<0$ then $f(x)$ is monotonically decreasing $\forall x>\beta$.

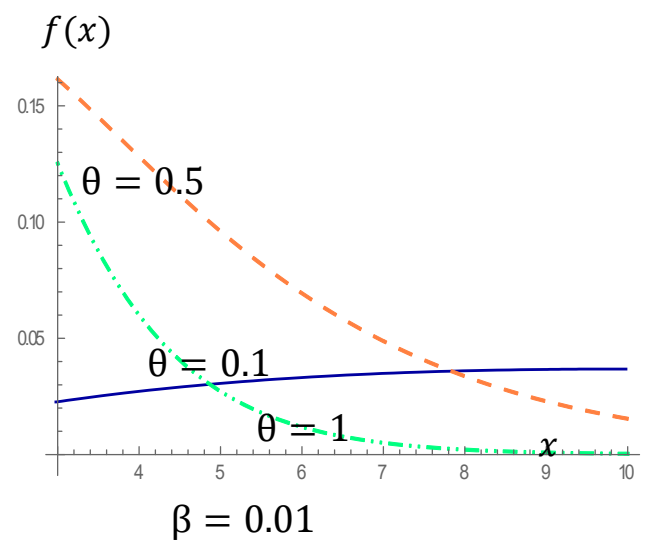

$$
\begin{aligned}
& f(x)_{\theta}=00039=5 \\
& f\left(x^{\theta}\right)=0.5 \\
& \beta=2 \\
& x^{x}
\end{aligned}
$$

$$
\beta=10
$$
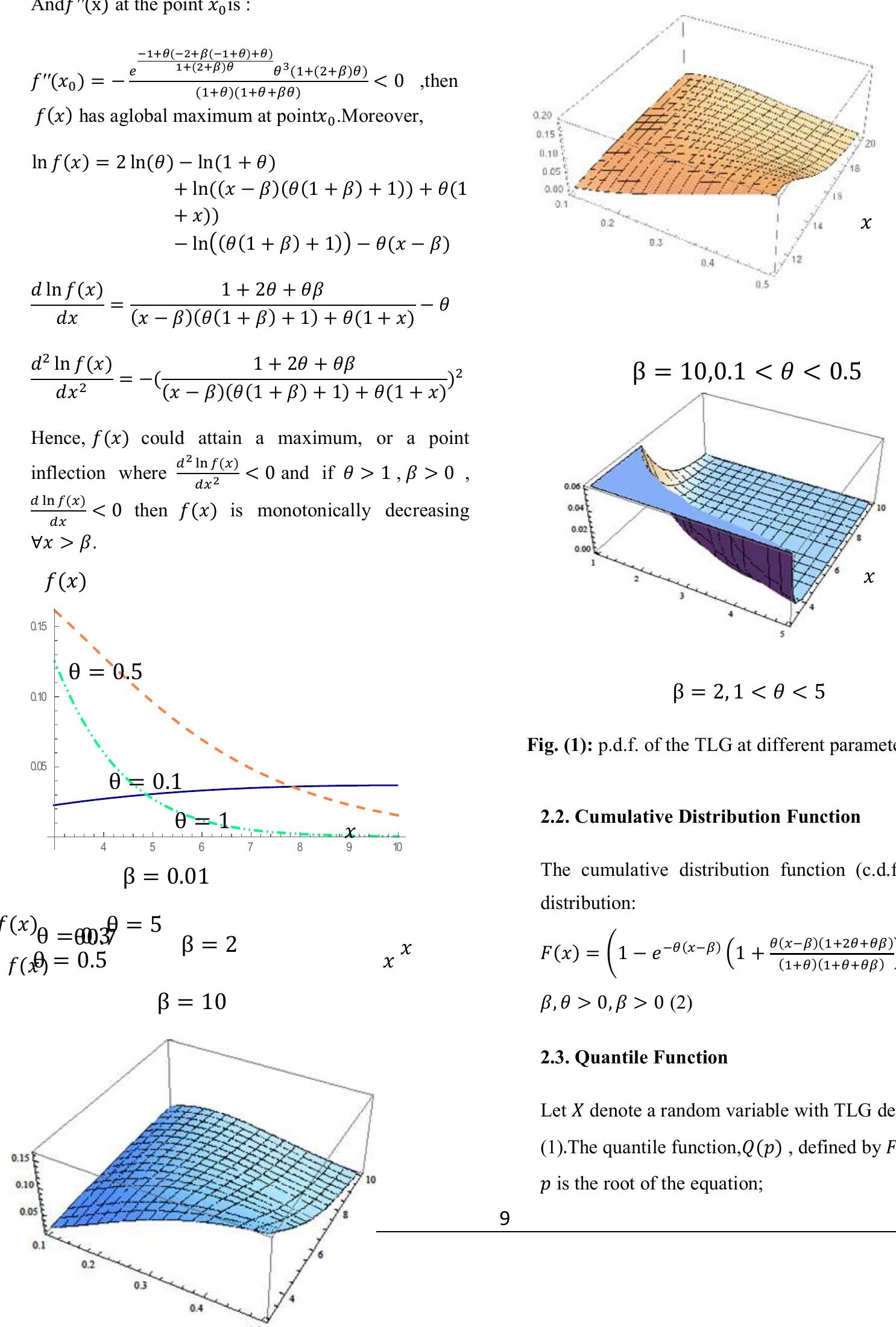

Fig. (1): p.d.f. of the TLG at different parameter values

\subsection{Cumulative Distribution Function}

The cumulative distribution function (c.d.f) of TLG distribution:

$F(x)=\left(1-e^{-\theta(x-\beta)}\left(1+\frac{\theta(x-\beta)(1+2 \theta+\theta \beta)}{(1+\theta)(1+\theta+\theta \beta)}\right)\right), x>$

$\beta, \theta>0, \beta>0(2)$

\subsection{Quantile Function}

Let $X$ denote a random variable with TLG density (1). The quantile function, $Q(p)$, defined by $F(Q(p))=$ $p$ is the root of the equation; 


$$
\begin{aligned}
Q(p)=(-1+\theta & (-2+(-1+\beta) \theta+\beta \theta \beta)-(1+2 \theta \\
& +\theta \beta) \text { ProductLog }\left[\left(e^{\wedge}(-((1+\theta)(1\right.\right. \\
& +\theta+\beta \theta)) /(1+2 \theta+\theta \beta))(-1 \\
& +P)(1+\theta)(1+\theta+\beta \theta)) /(1+2 \theta \\
& +\theta \beta)]) /(\theta(1+2 \theta+\theta \beta))
\end{aligned}
$$

\section{Reliability Measures}

\subsection{Survival function}

The reliability (survival) of TLG is defined by

$$
\begin{aligned}
S(x)=e^{-\theta(x-\beta)}\left(1+\frac{\theta(x-\beta)(1+2 \theta+\theta \beta)}{(1+\theta)(1+\theta+\theta \beta)}\right), x> \\
\beta, \theta>0, \beta>0
\end{aligned}
$$

\subsection{Hazard rate function}

The hazard (or failure) rate function is defined as $h(x)=\frac{f(x)}{S(x)}$

$$
h(x)=\frac{\theta^{2}(x+\theta+x(2+\beta) \theta-\beta(1+\theta+\beta \theta))}{1+\theta(2+x+\theta-\beta(1+\beta) \theta+x(2+\beta) \theta)}, \mathrm{x}>
$$

$\beta, \theta>0, \beta>0$ (4)

The behaviour of $h(x)$ at $x=\beta$ and at infinity respectively,is given by

$h(\beta)=f(\beta)=\frac{(1+\beta) \theta^{3}}{(1+\theta)(1+\theta+\beta \theta)} \quad, \quad \lim _{x \rightarrow \infty} h(x)=\theta$

\section{Theorem 2.}

The hazard rate function $h(x)$ of the TLG distribution is increasing

\section{Proof.}

Using Glaser[6], since $\eta(x)=-\frac{d}{d x} \ln f(x)=\frac{f^{\prime}(x)}{f(x)}=$ $-\frac{1+2 \theta+\theta \beta}{(x-\beta)(\theta(1+\beta)+1)+\theta(1+x)}+\theta$

It follows that; $\eta^{\prime}(x)=\left(\frac{1+2 \theta+\theta}{(x-\beta)(\theta(1+\beta)+1)+\theta(1+x)}\right)^{2}$

For $>0, \eta^{\prime}(x)>0$, i.e $\eta(x)$ is increasing . Therefore $h(x)$ is increasing Fig(2).

$h(x)$

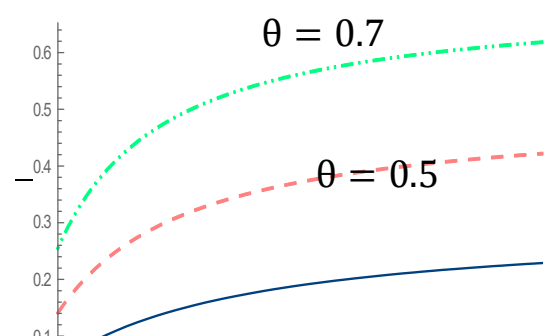

$$
\theta=0.3
$$

$$
\beta=10
$$

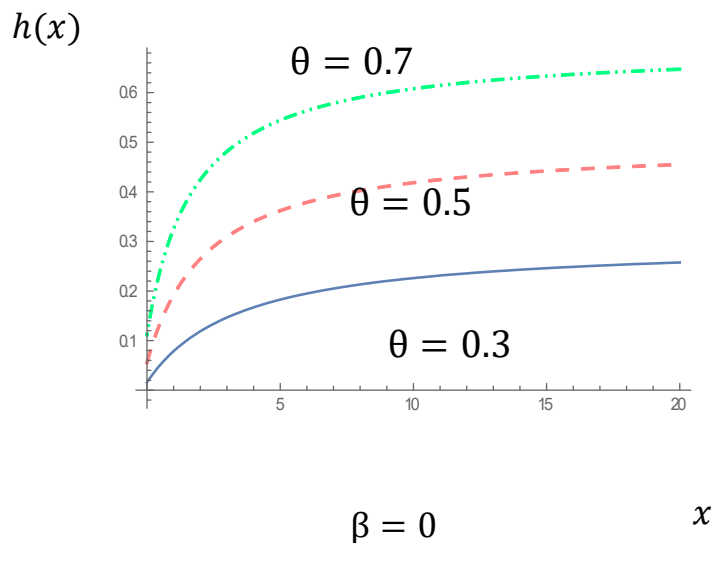

Fig. (2): Hazard function of the TLG at different parameter values

\subsection{Reversed hazard function}

Reversed hazard function is defined by $r(x)=\frac{f(x)}{F(x)}$, then for TLG distribution

$r(x)=$

$\frac{e^{(-x+\beta) \theta} \theta^{2}(x+\theta+x(2+\beta) \theta-\beta(1+\theta+\beta \theta))}{-(1+\theta)(1+\theta+\beta)+e^{(-x+\beta) \theta}(1+\theta(2+x+\theta-\beta(1+\beta) \theta+x(2+\beta) \theta))}, x>$ $\beta, \theta>0, \beta>0(5)$

Thereversed hazard rate function $r(x)$ of TLG distribution is decreasing.

$r(x)$

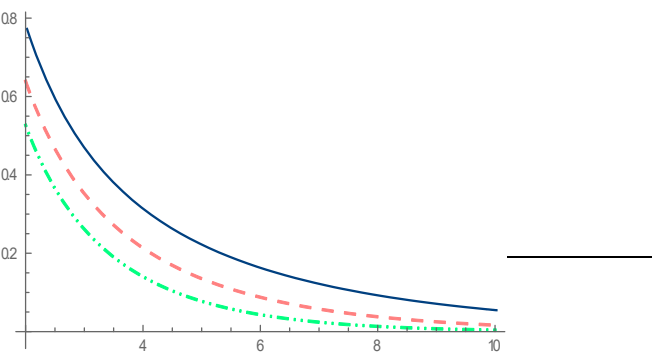




$$
\begin{gathered}
\theta=0.3, \beta=0.1 \\
\theta=0.5, \beta=0.1 \quad x
\end{gathered}
$$

Fig. (3): Reversedhazard function of the TLG at different parameter values

\subsection{Mean residual life function}

The mean residual life function $\mu(x)=E(X-x \mid \mathrm{X}>$ $\mathrm{x})$ of TLG distribution is defined by

$$
\begin{gathered}
\mu(\mathrm{x})=\frac{1}{R(x)} \int_{x}^{\infty} R(t) \mathrm{d} t \\
\frac{2(3+\theta(6+x+\theta+x(2+\beta) \theta-\beta(-2+\theta+\beta \theta)))}{\theta^{2}(1+\theta(2+x+\theta-\beta(1+\beta) \theta+x(2+\beta) \theta))}
\end{gathered}
$$

The behaviour of $\mu(\mathrm{x})$ at $x=0$ and at infinity, respectively, are driven by

$\mu(0)=\frac{2+\theta(4+\beta+\theta-\beta(1+\beta) \theta)}{\theta(1+\theta(2+\theta-\beta(1+\beta) \theta))}, \quad \lim _{x \rightarrow \infty} \mu(x)=\frac{1}{\theta}$

For TLG distribution the mean residual life $\mu(\mathrm{x})$ is decreasing Fig 4, since $h(x)$ isincreasing according to Lemma(2), Brysson and Siddique[3]

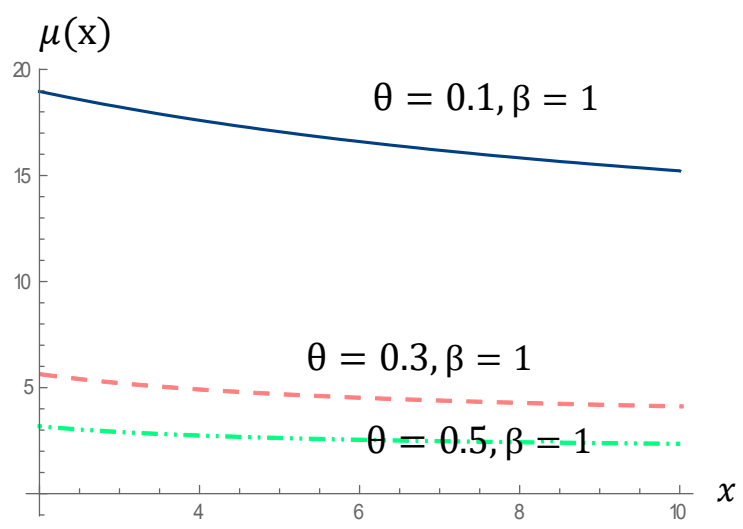

Fig(4). Mean residual life of TLG at different parameter values

\section{Moments and Associated Measures}

The $r^{\text {th }}$ raw moments (about the origin) of TLG distribution is given by

$$
\mu_{r}^{\prime}=E\left(x^{r}\right)=\int_{\beta}^{\infty} x^{r} f(x) \mathrm{d} x
$$

$\mu_{r}^{\prime}$

$=\int_{\beta}^{\infty} x^{r} \frac{\theta^{2}}{1+\theta}\left((x-\beta)+\frac{\theta(1+x)}{\theta(1+\beta)+1}\right) e^{-\theta(x-\beta)} \mathrm{d} x$

$=\frac{1}{(1+\theta)(1+\theta+\beta \theta)} \theta^{-r}(\beta \theta)^{-r}\left(\beta^{r} \theta^{r}(\beta \theta)^{1+r}(1\right.$

$+(2+\beta) \theta)+e^{\beta \theta}(1+r+r(2+\beta) \theta+\theta(2-(-1$

$\left.\left.\left.+\beta+\beta^{2}\right) \theta\right)\right)\left(r(\beta \theta)^{r} \Gamma[r]+\beta^{r} \theta^{r}(-\Gamma[1+r]+\Gamma[1\right.$

$+r, \beta \theta]))$ )

The first four moments about the origin of the TLG distribution have been obtained as:

$$
\begin{aligned}
& \mu_{1}^{\prime}=\frac{2+\theta(4+\theta}{\theta}+\beta(3+\theta(3+\beta+\theta+\beta \theta))) \\
& \mu_{2}^{\prime}=(1+\theta+\beta(2(6+\theta)+\beta(10+\theta(2(5+\theta)+\beta(5 \\
&+\theta(4+\beta+\theta+\beta \theta))))) /\left(\theta^{\wedge} 2(1\right. \\
&+\theta)(1+\theta+\beta \theta)) \\
& \mu_{3}^{\prime}=((24+\theta(6(8+\theta)+\beta(42+\theta(6(7+\theta) \\
&+\beta(24+\theta(3(6+\theta)+\beta(7+\theta(5 \\
&+\beta+\theta+\beta \theta)))))))) /\left(\left(\theta^{\wedge} 3(1\right.\right. \\
&+\theta)(1+\theta+\beta \theta))) \\
& \\
& \mu_{4}^{\prime}=((120+\theta(10+\theta)+\beta(216+\theta(24(9+\theta) \\
&+\beta(132+\theta(12(8+\theta)+\beta(44 \\
&+\theta(4(7+\theta)+\beta(9+\theta(6+\beta+\theta \\
&+\beta \theta))))))))))) /\left(\left(\theta^{\wedge} 4(1+\theta)(1\right.\right. \\
&+\theta+\beta \theta)))
\end{aligned}
$$

Therefore, the mean and variance of TLG distribution respectively, are

$\mu=\frac{2+\theta(4+\theta+\beta(3+\theta(3+\beta+\theta+\beta \theta)))}{\theta(1+\theta)(1+\theta+\beta \theta)}, \sigma^{2}=$

$\frac{2+4(2+\beta) \theta+2(6+\beta(6+\beta)) \theta^{2}+4(1+\beta)(2+\beta) \theta^{3}+(1+\beta)^{2} \theta^{4}}{\theta^{2}(1+\theta)^{2}(1+\theta+\beta \theta)^{2}}$

The first four central Moments about the mean are then given by:

$$
\begin{gathered}
\mu_{k}=E\left[(x-\mu)^{k}\right] \\
\mu_{2}=\left(2+4(2+\beta) \theta+2(6+\beta(6+\beta)) \theta^{\wedge} 2+4(1\right. \\
+\beta)(2+\beta) \theta^{\wedge} 3+\llbracket(1 \\
\left.+\beta) \rrbracket \wedge 2 \theta^{\wedge} 4\right) /\left(\theta^{\wedge} 2 \llbracket(1\right. \\
+\theta) \rrbracket \wedge 2 \llbracket(1+\theta+\beta \theta) \rrbracket \wedge 2)
\end{gathered}
$$

$$
\begin{aligned}
\mu_{3}=(2(2+6(2 & +\beta) \theta+6(5+\beta(5+\beta)) \theta^{2}+2(2 \\
& +\beta)(10+\beta(10+\beta)) \theta^{3}+6(1 \\
& +\beta)(5+\beta(5+\beta)) \theta^{4} \\
& +6(1+\beta)^{2}(2+\beta) \theta^{5} \\
& \left.\left.+(1+\beta)^{3} \theta^{6}\right)\right) \\
& /\left(\theta^{3}(1+\theta)^{3}(1+\theta+\beta \theta)^{3}\right)
\end{aligned}
$$




$$
\begin{aligned}
\mu_{4}=(3(8+32 & (2+\beta) \theta+16(14+\beta(14+3 \beta)) \theta^{2} \\
& +32(2+\beta)(7+\beta(7+\beta)) \theta^{3} \\
& +4(139+\beta(278+\beta(179 \\
& +2 \beta(20+\beta)))) \theta^{4}+8(1+\beta)(2 \\
& +\beta)(27+\beta(27+4 \beta)) \theta^{5} \\
& +4(1+\beta)^{2}(50+\beta(50+11 \beta)) \theta^{6} \\
& +24(1+\beta)^{3}(2+\beta) \theta^{7} \\
& \left.\left.+3(1+\beta)^{4} \theta^{8}\right)\right) \\
& /\left(\theta^{4}(1+\theta)^{4}(1+\theta+\beta \theta)^{4}\right)
\end{aligned}
$$

The skewness $\left(\beta_{1}\right)$ and kurtosis $\left(\beta_{2}\right)$ measures can be obtained from the expressions

$$
\begin{gathered}
\beta_{1}={ }_{A} \\
\frac{\beta_{2}=}{\left(2+4(2+\beta) \theta+2(6+\beta(6+\beta)) \theta^{2}+4(1+\beta)(2+\beta) \theta^{3}+(1+\beta)^{2} \theta^{4}\right)^{3}} \\
\frac{B}{\left(2+4(2+\beta) \theta+2(6+\beta(6+\beta)) \theta^{2}+4(1+\beta)(2+\beta) \theta^{3}+(1+\beta)^{2} \theta^{4}\right)^{2}}
\end{gathered}
$$

Where,

$$
\begin{aligned}
A=(4(2+6(2 & +\beta) \theta+6(5+\beta(5+\beta)) \theta^{2}+2(2 \\
& +\beta)(10+\beta(10+\beta)) \theta^{3}+6(1 \\
& +\beta)(5+\beta(5+\beta)) \theta^{4} \\
& +6(1+\beta)^{2}(2+\beta) \theta^{5} \\
& \left.\left.+(1+\beta)^{3} \theta^{6}\right)^{2}\right) \\
B=(3(8+32(2 & +\beta) \theta+16(14+\beta(14+3 \beta)) \theta^{2} \\
& +32(2+\beta)(7+\beta(7+\beta)) \theta^{3} \\
& +4(139+\beta(278+\beta(179 \\
& +2 \beta(20+\beta)))) \theta^{4}+8(1+\beta)(2 \\
& +\beta)(27+\beta(27+4 \beta)) \theta^{5} \\
& +4(1+\beta)^{2}(50+\beta(50+11 \beta)) \theta^{6} \\
& +24(1+\beta)^{3}(2+\beta) \theta^{7} \\
& \left.\left.+3(1+\beta)^{4} \theta^{8}\right)\right)
\end{aligned}
$$

\section{The Characteristic and the Moment generating functions}

The characteristic function of TLG distribution is given by

$$
\begin{array}{r}
\varphi(t)=\int_{\beta}^{\infty} e^{i t x}\left(\frac{\theta^{2}}{1+\theta}((x-\beta)\right. \\
\left.\left.\quad+\frac{\theta(1+x)}{\theta(1+\beta)+1}\right) e^{-\theta(x-\beta)}\right) \mathrm{d} x \\
=\frac{e^{i t \beta} \theta^{2}(1+\theta(2+\beta-i t(1+\beta)+\theta+\beta \theta))}{(1+\theta)(-i t+\theta)^{2}(1+\theta+\beta \theta)}
\end{array}
$$

The moment generating function of TLG distribution is given by

$$
\begin{array}{r}
M_{x}(t)=\int_{\beta}^{\infty} e^{t x}\left(\frac{\theta^{2}}{1+\theta}((x-\beta)\right. \\
\left.\left.\quad+\frac{\theta(1+x)}{\theta(1+\beta)+1}\right) e^{-\theta(x-\beta)}\right) \mathrm{d} x \\
=\frac{e^{t \beta} \theta^{2}(1+\theta(2+\beta-t(1+\beta)+\theta+\beta \theta))}{(t-\theta)^{2}(1+\theta)(1+\theta+\beta \theta)},
\end{array}
$$

\section{Bonferroni and Lorenz Curves}

The Bonferroni and Lorenz curves and Gini index have many applications not only in economics to study income and poverty, but also in other fields like reliability, medicine and insurance .

The Bonferroni curve $B_{F}[F(x)]$ is given by

$B_{F}[F(x)]=\frac{1}{\mu F(x)} \int_{0}^{u} u f(u) d u$

Equivalently given by

$B_{F}(p)=\frac{1}{\mu p} \int_{0}^{p} F^{-1}(t) d t$

where $p=F(x)$ and $F^{-1}(t)=\inf \{x: F(x) \geq t\}$.From the relationship between the Bonferroni curve and the mean residual life lifetime given by theorem 2.1 of Pundir et al.[13], the Bonferroni curve of distribution function F of TLG distribution is given by

$$
\begin{aligned}
B_{F}[F(x)]=1 /( & ((2+\theta(4+\theta+\beta(3+\theta(3+\beta+\theta \\
& +\beta \theta)))) /(\theta(1+\theta)(1+\theta \\
& +\beta \theta)))\left(1-\left(e^{\wedge}((\beta-x) \theta)(1\right.\right. \\
& +\theta(2+x+\theta-\beta(1+\beta) \theta+x(2 \\
& +\beta) \theta))) /((1+\theta)(1+\theta \\
& +\beta \theta)))) \int \beta^{\wedge} x \text { w } \llbracket \theta^{\wedge} 2 /(1 \\
& +\theta)((u-\beta)+(\theta(1+u)) /(\theta(1 \\
& +\beta)+1)) e^{\wedge}(-\theta(u-\beta)) \mathrm{d} u \rrbracket \\
B_{F}[F(x)]=\mathrm{C} /( & \left(\left((1+\theta)(1+\theta+\beta \theta)-e^{\wedge}((-x\right.\right. \\
& +\beta) \theta)(1+\theta(2+x+\theta-\beta(1 \\
& +\beta) \theta+x(2+\beta) \theta))(2+\theta(4+\theta \\
& +\beta(3+\theta(3+\beta+\theta+\beta \theta)))))
\end{aligned}
$$

Where, 


$$
\begin{aligned}
C=((1+\theta)(1+ & \theta+\beta \theta)(2+\theta(4+\theta+\beta(3+\theta(3 \\
& +\beta+\theta+\beta \theta)))-e^{(-x+\beta) \theta}(2 \\
& +\theta(4+\beta+\theta-\beta(1+\beta) \theta \\
& +x^{2} \theta(1+(2+\beta) \theta)+x(2+\theta(4 \\
& +\beta+\theta-\beta(1+\beta) \theta))))))
\end{aligned}
$$

In addition, Lorenz curve of $\mathrm{F}$ that follows the TLG distribution can be obtained by $\quad L_{F}[F(x)]=$ $B_{F}[F(x)] F(x)$.

The scaled total time and cumulative total time on test transform of a distribution function FPundir et al.[13] are respectively obtained by,

$S_{F}[F(t)]=\frac{1}{\mu} \int_{0}^{t} \bar{F}(u) d u, C_{F}=\int_{0}^{1} S_{F}[F(t)] f(t) d t$

Hence, for TLG distribution

$$
\begin{aligned}
S_{F}[F(t)]=(2+ & \theta(4+\theta+\beta(2+\theta))-e^{\wedge}((-t \\
& +\beta) \theta)(2+\theta(4+\beta+\theta-\beta(1 \\
& +\beta) \theta+t(1+(2+\beta) \theta)))) /(2 \\
& +\theta(4+\theta+\beta(3+\theta(3+\beta+\theta \\
& +\beta \theta)))) \\
C_{F}=\mathrm{D} /\left(\left(\theta^{\wedge} 2(1+\right.\right. & \theta+\beta \theta)\left(\left(1-\left(e^{\wedge}((-x\right.\right.\right. \\
& +\beta) \theta)(1+\theta(2+x+\theta-\beta(1 \\
& +\beta) \theta+x(2+\beta) \theta))) /((1+\theta)(1 \\
& +\theta+\beta \theta)))(2+\theta(4+\theta+\beta(3 \\
& +\theta(3+\beta+\theta+\beta \theta))))) \times \theta^{\wedge} 2 /(1 \\
& +\theta)))
\end{aligned}
$$

Where, $D=\left(\left((1+\theta)(1+\theta+\beta \theta)+e^{(-1+\beta) \theta}(-1+\right.\right.$ $\left.\left.\theta\left(-3+\left(-3+\beta^{2}\right) \theta\right)\right)\right)(2+\theta(4+\theta+\beta(3+\theta(3+$ $\beta+\theta+\beta \theta)))-e^{(-x+\beta) \theta}(2+\theta(4+\beta+\theta-\beta(1+$ $\beta) \theta+x^{2} \theta(1+(2+\beta) \theta)+x(2+\theta(4+\beta+\theta-$ $\beta(1+\beta) \theta)))))($

The Gini index can be obtained from the relationship = $1-C_{F}$.

\section{Estimation and inference}

In this section, we consider maximum likelihood estimation and method of moment estimation. In addition, providing expressions for the associated observed Fisher information matrix

\subsection{Maximum likelihood estimation}

Let $x_{1}, x_{2}, \ldots \ldots, x_{n}$ be a random sample of size $\mathrm{n}$ from the TLG distribution with p.d.f (1). The log-likelihood function $\mathcal{L}(\theta, \beta)$ is given by

$$
\begin{aligned}
& \mathcal{L}(\theta, \beta)=2 \mathrm{n} \log \theta-\mathrm{n} \log (1+\theta)+\sum_{\mathrm{i}=1}^{\mathrm{n}} \log \left(\left(\mathrm{x}_{\mathrm{i}}-\beta\right)\right. \\
&\left.+\frac{\theta\left(1+\mathrm{x}_{\mathrm{i}}\right)}{\theta(1+\beta)+1}\right)-\theta \sum_{\mathrm{i}=1}^{\mathrm{n}} \mathrm{x}_{\mathrm{i}}+\theta \mathrm{n} \beta
\end{aligned}
$$

It follows that the maximum likelihood estimators (MLEs), say $\hat{\theta}, \hat{\beta}$, are the simultaneous solutions of the equations,

$U_{1}(\theta, \beta)=\frac{\partial}{\partial \theta} \mathcal{L}(\theta, \beta)$

$=n \beta-\sum_{i=1}^{n} x_{i}$

$+\sum_{i=1}^{n} \frac{1+x_{i}}{(1+\theta+\beta \theta)\left(\theta-\beta(1+\theta+\beta \theta)+(1+(2+\beta) \theta) x_{i}\right)}$

$+\frac{2 n}{\theta}-\frac{n}{1+\theta}$

$\hat{\beta}=\min x_{i}, \quad 1 \leq i \leq n$

\subsection{Method of Moments}

The method of moments is another commonly technique used in parameter estimation For the TLG distribution, we have

$$
\begin{aligned}
& E(X \mid \theta, \beta)=\frac{2+\theta(4+\theta+\beta(3+\theta(3+\beta+\theta+\beta \theta)))}{\theta(1+\theta)(1+\theta+\beta)} \\
& \operatorname{Var}(X \mid \theta, \beta)=\left(\begin{array}{l}
6 \\
+\theta(2(6+\theta)+\beta(10+\theta(2(5 \\
+\theta)+\beta(5+\theta(4+\beta+\theta \\
+\beta \theta)))))) /\left(\theta^{\wedge} 2(1+\theta)(1+\theta\right. \\
+\beta \theta))
\end{array}\right.
\end{aligned}
$$

The method of moments estimates $\hat{\theta}_{M M E}$ and $\hat{\beta}_{M M E}$ for $\theta$ and $\beta$ respectively, are obtained by solving the equations :

$E\left(X \mid \hat{\theta}_{M M E}, \hat{\beta}_{M M E}\right)=\bar{x}, \quad \operatorname{Var}\left(X \mid \hat{\theta}_{M M E}, \hat{\beta}_{M M E}\right)=$ $S^{2}$

\subsection{Information matrix}


The elements of the expected Fisher information matrix $=\left[I_{i j}\right], i, j=1,2$, about $(\theta, \beta)$ from a single observation are given by

$$
\begin{aligned}
& I_{11}=E\left[-\frac{\partial}{\partial \theta^{2}} \ln f(x)\right] \\
& I_{22}=E\left[-\frac{\partial}{\partial \beta^{2}} \ln f(x)\right] \\
& I_{12}=I_{21}=E\left[-\frac{\partial^{2}}{\partial \theta \partial \beta} \ln f(x)\right] \\
& {\left[\frac{\partial}{\partial \theta^{2}} \ln f(x)\right]} \\
& =-\frac{2}{\theta^{2}}+\frac{1}{(1+\theta)^{2}}+\frac{(1+\beta)^{2}}{(1+\theta+\beta \theta)^{2}} \\
& -\frac{\left(-1+\beta+\beta^{2}-x(2+\beta)\right)^{2}}{(x+\theta+x(2+\beta) \theta-\beta(1+\theta+\beta \theta))^{2}} \\
& {\left[\frac{\partial}{\partial \beta^{2}} \ln f(x)\right]} \\
& =\frac{-1+\frac{(1+x) \theta^{2}(-2+\theta(-4+2 x-6 \beta-\theta+(x-2 \beta)(3+2 \beta) \theta))}{(1+\theta+\beta)^{4}}}{\left(x-\beta+\frac{(1+x) \theta}{1+\theta+\beta}\right)^{2}} \\
& {\left[\frac{\partial^{2}}{\partial \theta \partial \beta} \ln f(x)\right]} \\
& =1-\frac{1}{(1+\theta+\beta \theta)^{2}} \\
& +\frac{1+x+x^{2}-2 x \beta+\beta^{2}}{(x+\theta+x(2+\beta) \theta-\beta(1+\theta+\beta \theta))^{2}}
\end{aligned}
$$

Applying standard large-sample theory of maximum likelihood estimators, Lehmann et al.[8] we have

$$
\sqrt{n}\left(\begin{array}{l}
\hat{\theta}-\theta \\
\hat{\beta}-\beta
\end{array}\right) \stackrel{d}{\rightarrow} N_{2}\left(0, I^{-1}\right),
$$

Where $\stackrel{d}{\rightarrow}$ denotes convergence in distribution and $I^{-1}$ is the inverse of the matrix $I$.

Now the asymptotic variances and covariance of MLEs $\hat{\theta}$ and $\hat{\beta}$ are given by

$\operatorname{var}(\hat{\theta})=\frac{I_{22}}{n \Delta}, \operatorname{var}(\hat{\beta})=\frac{I_{11}}{n \Delta}, \operatorname{cov}(\hat{\theta}, \hat{\beta})=\frac{-I_{12}}{n \Delta}$

where $\Delta=I_{11} I_{22}-I_{12}^{2}$ is the determinant of the matrix I .

The asymptotic $100(1-\alpha) \%$ confidence interval of $\theta$ and $\beta$, respectively, are given by, $\hat{\theta} \pm z \alpha / 2 \sqrt{\widehat{\operatorname{var}(\hat{\theta})}}$ $\hat{\theta} \pm Z \alpha / 2 \sqrt{\operatorname{var(\hat {\beta })}}$ where $\sqrt{\widehat{\operatorname{var}(\hat{\theta})}}, \sqrt{\widehat{\operatorname{var}(\hat{\beta})}}$ is the MLE of $\operatorname{var}(\hat{\theta}), \operatorname{var}(\hat{\beta})$ and $z \alpha / 2$ is the upper $\alpha / 2$ quantile of the standard normal distribution.

\section{Probability and Cumulative Function of Order Statistics}

Suppose $X_{1}, X_{2}, \ldots \ldots, X_{n}$ is a random sample from TLG distribution. Let $X_{1: n}<X_{2: n}<\cdots<X_{n: n}$ denote the corresponding order statistics. The probability density function and the cumulative distribution function of the $k t h$ order statistic, say $Y=X_{k: n}$ are given by

$$
\begin{gathered}
f_{Y}(y)=\frac{n !}{(k-1) !(n-k) !} F^{k-1}(y)\{1 \\
-F(y)\}^{n-k} f(y)
\end{gathered}
$$

$f_{Y}(y)$

$$
\begin{aligned}
& =\frac{n !}{\Gamma[k] \Gamma[1-k+n]} \cdot\left[\frac{\theta^{2}}{1+\theta}((y-\beta)\right. \\
& \left.\left.+\frac{\theta(1+y)}{\theta(1+\beta)+1}\right) e^{-\theta(y-\beta)}\right] \cdot[1 \\
& -e^{-\theta(y-\beta)}(1 \\
& \left.\left.+\frac{\theta(y-\beta)(1+2 \theta+\theta \beta)}{(1+\theta)(1+\theta+\theta \beta)}\right)\right]^{k-1} \cdot\left[e^{-\theta(y-\beta)}(1\right. \\
& \left.\left.+\frac{\theta(y-\beta)(1+2 \theta+\theta \beta)}{(1+\theta)(1+\theta+\theta \beta)}\right)\right]^{n-k}
\end{aligned}
$$

And,

$$
\begin{aligned}
& F_{Y}(y)=\sum_{m=k}^{n}\left(\begin{array}{l}
n \\
m
\end{array}\right) F^{m}(y) \times[1-F(y)]^{n-m} \\
& F_{Y}(y)=\sum_{m=k}^{n}\left(\begin{array}{c}
n \\
m
\end{array}\right)(1 \\
&-e^{-\theta(y-\beta)}(1 \\
&+\left.\left.\frac{\theta(y-\beta)(1+2 \theta+\theta \beta)}{(1+\theta)(1+\theta+\theta \beta)}\right)\right)^{m} \\
& \times\left[e^{-\theta(y-\beta)(1}\right. \\
&\left.\left.+\frac{\theta(y-\beta)(1+2 \theta+\theta \beta)}{(1+\theta)(1+\theta+\theta \beta)}\right)\right]^{n-m}
\end{aligned}
$$

\section{Simulation Studies}

The equation $(x)-u=0$, where $\mathrm{u}$ is an observation from the uniform distribution on $(0,1)$ and $F(x)$ is cumulative distribution function of distribution is used to carry out the simulation study to generate data from distribution. The simulation experiment was repeated 
$N=1000$ times each with sample sizes; $n=$ $30,50,70,90$ and $(\theta, \beta)=(1,1),(0.1,0.01),(1,0.7)$

.The following measures were computed:

(i) Average bias of $\hat{\gamma}$ of the parameter $\theta, \beta$

$$
\frac{1}{N} \sum_{i=1}^{N}(\hat{\gamma}-\gamma)
$$

(ii) The Mean square error (MSE) of the $\hat{\gamma}$ of the parameter $\theta, \beta$

$$
\frac{1}{N} \sum_{i=1}^{N}(\hat{\gamma}-\gamma)^{2}
$$

Table (1) present the average bias and the MSE of the estimates. The values of the bias are seen to be small and positive and the values of the MSE's decreases while the sample size increases.

Table (1) :Bias and MSE for the parameters $\theta, \beta$

\begin{tabular}{|c|c|c|c|c|c|c|}
\hline $\boldsymbol{\theta}$ & $\boldsymbol{\beta}$ & $\mathrm{N}$ & $\begin{array}{c}\text { Bias } \\
(\boldsymbol{\theta})\end{array}$ & $\operatorname{MSE}(\boldsymbol{\theta})$ & $\operatorname{Bias}(\boldsymbol{\beta})$ & $\operatorname{MSE}(\boldsymbol{\beta})$ \\
\hline 1 & 1 & 30 & 0.0612 & 0.0483 & 0.0399 & 0.0808 \\
\hline \multirow{2}{*}{} & 50 & 0.0463 & 0.0259 & 0.0375 & 0.0529 \\
\cline { 3 - 7 } & 70 & 0.0355 & 0.0194 & 0.0333 & 0.0389 \\
\cline { 3 - 7 } & 90 & 0.0189 & 0.0137 & 0.0152 & 0.0275 \\
\hline 0.1 & 0.01 & 30 & 0.0063 & 0.0005 & 0.4484 & 10.3194 \\
\hline \multirow{3}{*}{1} & 50 & 0.0048 & 0.0003 & 0.4146 & 6.7339 \\
\cline { 3 - 7 } & 70 & 0.0037 & 0.0002 & 0.3784 & 4.9484 \\
\cline { 3 - 7 } & 90 & 0.0019 & 0.0001 & 0.1726 & 3.444 \\
\hline \multirow{2}{*}{0.7} & 30 & 0.0608 & 0.0480 & 0.0398 & 0.0846 \\
\hline & 50 & 0.0461 & 0.0258 & 0.0377 & 0.0553 \\
\cline { 3 - 7 } & 70 & 0.0354 & 0.0193 & 0.0336 & 0.0406 \\
\cline { 3 - 7 } & 90 & 0.0189 & 0.0136 & 0.0152 & 0.0287 \\
\hline
\end{tabular}

\section{Application}

This Section is devoted to illustrate the applicability of TLG distribution to real dataset represent the strength of glass of the aircraft window. The data is presented in Table (2) of Fuller[4] and Singh,et.al.[15].TLG distribution is fitted to these data and compared its fitting with some usual survival distributions. Namely, Weibull, Exponential, Gamma, Lindley(LD) and its truncated models (LTLD,RTLD), Burr, Logistic, Loglogistic and Frechet distributions.
Table 2: Strength of glass of the aircraft window

\begin{tabular}{|l|l|l|l|}
\hline 18.83 & 20.80 & 21.657 & 23.03 \\
\hline 23.23 & 24.05 & 24.321 & 25.5 \\
\hline 25.52 & 25.80 & 26.69 & 26.77 \\
\hline 26.78 & 27.05 & 27.67 & 29.9 \\
\hline 31.11 & 33.20 & 33.73 & 33.76 \\
\hline 33.89 & 34.76 & 35.75 & 35.91 \\
\hline 36.98 & 37.08 & 37.09 & 39.58 \\
\hline 44.045 & 45.29 & 45.381 & \\
\hline
\end{tabular}

To compare the goodness-of-fit of above models, we used the Akaikes information criterion (AIC), Corrected Akaikes information criterion (AICC), Bayesian information criterion (BIC) and KolmogorovSmirnov (K-S) statistic, which are calculated form the following formulae

$$
\begin{gathered}
A I C=-2 \log (L)+2 k \\
B I C=-2 \log (L)+k \log (n) \\
A I C C=A I C+\frac{2 k(k+1)}{(n-k-1)}
\end{gathered}
$$

where, $k$ is the number of parameters, $\mathrm{n}$ is the sample size. Based on the data, the fitting summary including the MLE's estimates of the parameters, log-likelihood, $A I C, A I C C, B I C$ and $K S$ statistics values have been summarized in Tables(3), indicate that TLG distribution is a good competitor to other commonly used in reliability field. Moreover, being the best fitting considering AIC, BIC, -Log and K-S criterion. 
Table 3 : Fitted estimates for different distributions

\begin{tabular}{|c|c|c|c|c|c|c|}
\hline $\begin{array}{c}\text { Distributi } \\
\text { on }\end{array}$ & Estimates & Log & AIC & AIC & BIC & K-S \\
\hline Gamma ( & $\hat{\alpha}=4.5394$ & 461.0 & 928.1 & 929.0 & 932.4 & 0.129 \\
$\alpha, \beta, \gamma)$ & $\hat{\beta}=3.4844$ & 84 & 68 & 5 & 7 & 64 \\
& $\hat{\gamma}=14.994$ & & & & & \\
\hline Burr & $\hat{\alpha}=6.1986$ & 310.6 & 627.2 & 628.1 & 631.5 & 0.139 \\
$((\alpha, \beta, k)$ & $\hat{\beta}=34.007$ & 11 & 22 & 11 & 24 & 61 \\
& $\hat{\kappa}=1.7253$ & & & & & \\
\hline LogLogisti & $\hat{\alpha}=4.6348$ & 263.0 & 526.0 & 526.9 & 5363 & 0.135 \\
c( $\alpha, \beta, \gamma)$ & $\hat{\beta}=19.26$ & 26 & 51 & 40 & 53 & 87 \\
& $\hat{\gamma}=10.442$ & & & & & \\
\hline Frechet( & $\hat{\alpha}=4.8652$ & 105.3 & 214.7 & 215.1 & 217.6 & 0.167 \\
$\alpha, \beta)$ & $\hat{\beta}=26.502$ & 84 & 68 & 96 & 36 & 57 \\
\hline Exp $(\lambda, v)$ & $\hat{\lambda}=0.08346$ & 107.9 & 219.9 & 220.3 & 222.8 & 0.201 \\
& $\hat{v}=18.83$ & 84 & 68 & 97 & 36 & 09 \\
\hline LD $(\theta)$ & $\hat{\theta}=0.06299$ & 126.9 & 225.9 & 256.1 & 256.9 & 0.333 \\
& & 94 & 88 & 26 & 71 & \\
\hline RTLD( & $\hat{\theta}=0.00754$ & 110.2 & 224.4 & 224.8 & 223.4 & 0.184 \\
$\theta, \xi)$ & $\hat{\xi}=45.381$ & 16 & 31 & 60 & 14 & \\
\hline Weibull( & $\hat{\alpha}=0.00017$ & 113.0 & 230.1 & 230.5 & 229.1 & 0.225 \\
$\alpha, \beta)$ & $\hat{\beta}=2.5046$ & 67 & 35 & 63 & 17 & \\
\hline LTLD( & $\hat{\theta}=0.10974$ & 107.1 & 218.2 & 218.6 & 217.1 & 0.15 \\
$\theta, v)$ & $\hat{v}=18.83$ & 03 & 05 & 34 & 88 & \\
\hline TLG $(\theta, \beta)$ & $\hat{\theta}=0.191$ & 104.4 & 212.8 & 213.2 & 215.7 & 0.120 \\
& $\hat{\beta}=0.894$ & 2 & 4 & 69 & 08 & 83 \\
\hline
\end{tabular}

Figure (5) illustrates the Q-Q plot for the considered dataset for all distributions considered in this paper. From the above results, it is evident that that mixture of truncated reliability models in the for of TLG distribution could provide better distribution for fitting the reliability dataset compared with other distributions considered here.
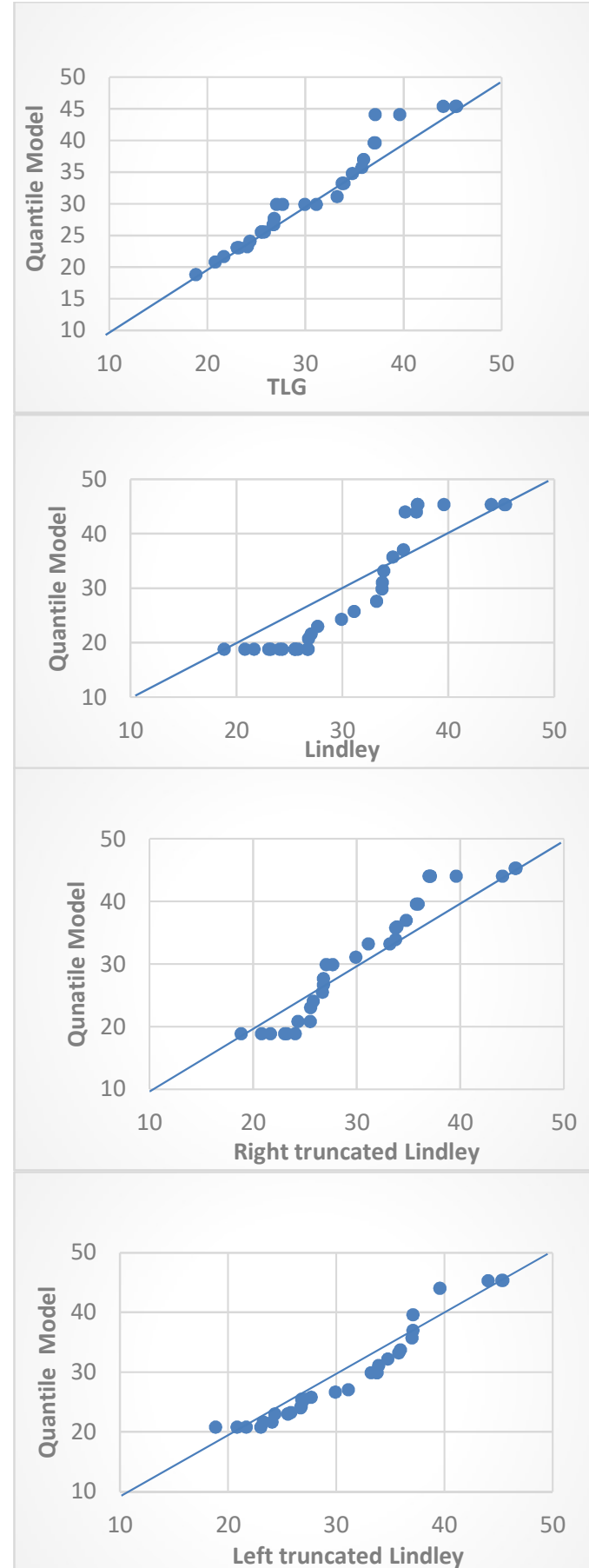

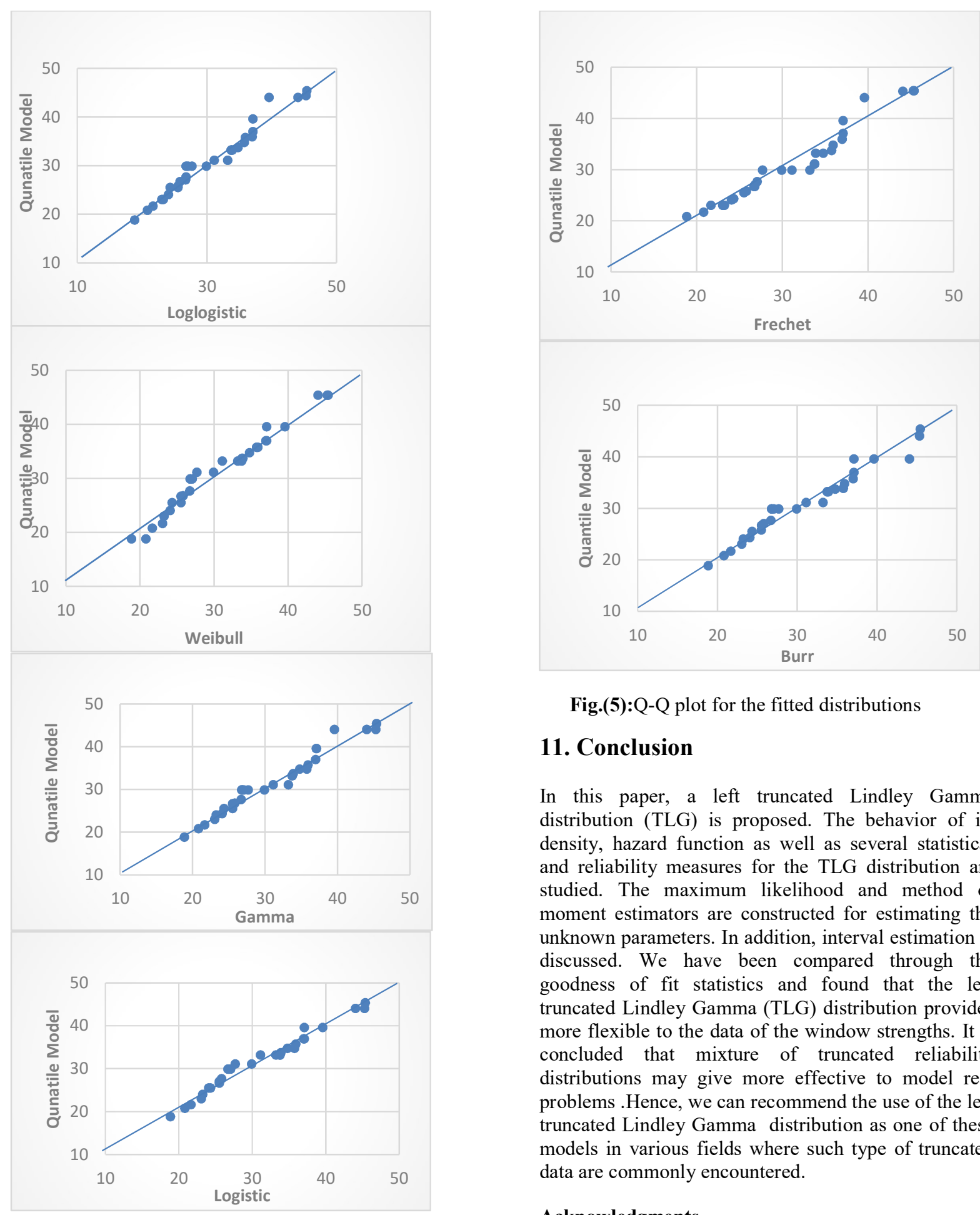

Fig.(5):Q-Q plot for the fitted distributions

\section{Conclusion}

In this paper, a left truncated Lindley Gamma distribution (TLG) is proposed. The behavior of its density, hazard function as well as several statistical and reliability measures for the TLG distribution are studied. The maximum likelihood and method of moment estimators are constructed for estimating the unknown parameters. In addition, interval estimation is discussed. We have been compared through the goodness of fit statistics and found that the left truncated Lindley Gamma (TLG) distribution provides more flexible to the data of the window strengths. It is concluded that mixture of truncated reliability distributions may give more effective to model real problems. Hence, we can recommend the use of the left truncated Lindley Gamma distribution as one of these models in various fields where such type of truncated data are commonly encountered.

\section{Acknowledgments}

The authors are very appreciative and thankful for Dr. Mohamed Mohamed Ezzat Abd El-Monsef for his valuable support in the application part, which enhanced the benefit of the paper.

\section{References}

[1] Aban, I. B. Meerschaert, M.M. Panorska, A. K. (2006). "Parameter estimation for the truncated Pareto 
distribution", Journal of the American Statistical Association (101) 270-277.

[2] Ahmed, S. E. Castro-Kuriss, C. Flores, E. Leiva, V. Sanhueza, A. (2010). "A truncated version of the birnbaum-saunders distribution with an application in financial risk", Pak. J. Statist. ( 26) 293-311.

[3] Bryson, M.C. Siddique, M.M. (1969). "Some criteria for aging", Journal of the American Statistical Association, (64) 1472-1483.

[4] Fuller-Jr, E. Frieman, S. Quinn, J. Quinn, G. Carter, W. (1994). "Fracture mechanics approach to the design of glass aircraft windows: A case study", SPIE Proc (22860), 419-430.

[5] Ghitany, M.E.; Atieh, B.; and Nadarajah, S. (2008). "Lindley Distribution and its Application". Mathematics and Computers in Simulation, 78: 493506.

[6] Glaser, R.E. (1980) "Bathtub and related failure rate characterization", Journal of the American Statistical Association, ( 75) 667-672.

[7]Lawless, J.F. (1982), "Statistical Models and Methods for Lifetime Data", Wiely, New York.

[8] Lehman, L.E. Casella, G. (1998) "Theory of Point Estimation", 2nd ed., Springer, NY.

[9] Lindley, D.V. ( 1965) “Introduction to Probability and Statistics from a Bayesian Viewpoint", Part II: Inference, Camb. Univ. Press, New York.

[10] Lindley, D.V. (1958). "Fiducial Distributions and Bayes' Theorem". Journal of the Royal Statistical Society, Series B, 20(1): 102-107.
[11] Mann, N.R. Schafer, R.E. Singpurwala, N.D. (1974), "Methods of Statistical Analysis of Reliability and Life Data", John Wiley \& Sons, New York.

[12] Murthy, D. P. Xie, M. R. Jiang, 2004, "Weibull Models", John Wiley \& Sons.

[13] Pundir, S.Arora ,S.,\&Jain,K.(2005). "Bonnferroni curve and the related statistical inference". Statistics and Probability Letters, 75(2),140-150.

[14] Sankaran, M. (2009), "The discrete PoissonLindley distribution", Test (18) 497-515.

[15] Singh, S. K.Singh, U. Sharma, V.K. (2014), "The Truncated Lindley Distribution: Inference and Application", Journal of Statistics Applications \& Probability.

[16] Sinha, S.K. (1986), "Reliability and Life Testing", Wiley Estern Limited, New Delhi.

[17] Zaninetti, L. Ferraro, M. (2008), “On the truncated Pareto distribution with applications", Central European Journal of Physics (6) 1-6.

[18] Zhang, T. Xie, M. (2011), "On the upper truncated Weibull distribution and its reliability implications", Reliability Engineering and System Safety ( 96) 194200.

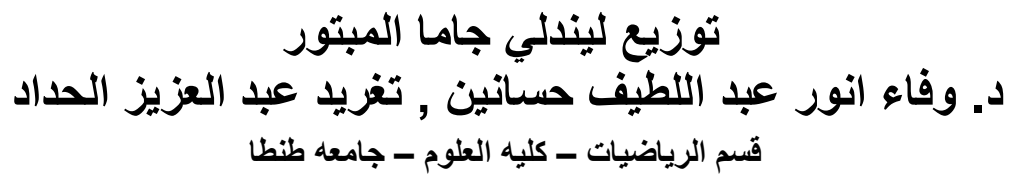

تم تقديم التوزيع الجديد ذات المعلمتين ليندلي جامـا المبتور ودراسه خصائصه ـ تضمن هذه الخصائص سلوك داله كثافه الاحتمال الخاصه بهذا التوزيع وداله المخاطره والعزوم المرتبطه وداله المخاطره العكسيه ومتوسط العمر المتبقي ومنحنيات لورينز وبنفروني. التقدير بنقطه بإستخدام دالة الإمكان العظمي وطريقه العزوم. علاوة علي ذلك تم تحقيق التقدير بفترة ـ تم

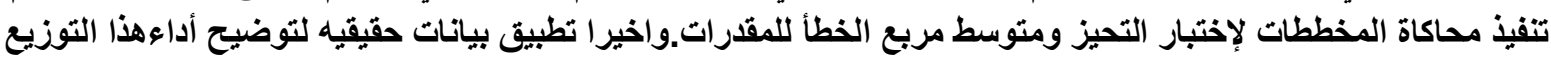
ومقارنته ببعض التوزيعاث المعروفه. 